Article

\title{
Effects of Poly(cyclohexanedimethylene terephthalate) on Microstructures, Crystallization Behavior and Properties of the Poly(ester ether) Elastomers
}

\author{
Yi-Cheng Feng ${ }^{1}$, Hui Zhao ${ }^{1}$, Tong-Hui Hao ${ }^{1}$, Guo-Hua Hu ${ }^{2}$, Tao Jiang ${ }^{1}$ and \\ Qun-Chao Zhang ${ }^{1, *}$ \\ 1 Hubei Collaborative Innovation Center for Advanced Organic Chemical Materials, Ministry of Education \\ Key Laboratory of Green Preparation and Application for Functional Materials, School of Materials Science \\ \& Engineering, Hubei University, Wuhan 430062, China; fengyicheng0221@icloud.com (Y.-C.F.); \\ zhh0625@foxmail.com (H.Z.); haoth@hubu.edu.cn (T.-H.H.); jiangtao@hubu.edu.cn (T.J.) \\ 2 Laboratory of Reactions and Process Engineering, CNRS-University of Lorraine, 1 rue Grandville, BP 20451, \\ 54001 Nancy Cedex, France; guo-hua.hu@univ-lorraine.fr \\ * Correspondence: zhangqc1976@hubu.edu.cn; Tel.: +86-158-7128-3888
}

Received: 17 May 2017; Accepted: 22 June 2017; Published: 24 June 2017

\begin{abstract}
To understand the role of molecular structure on the crystallization behavior of copolyester in thermoplastic poly(ether ester) elastomers (TPEEs), series of poly(butylene-co-1,4-cyclohexanedimethylene terephthalate) (P(BT-co-CT))-b-poly(tetramethylene glycol) (PTMG) are synthesized through molten polycondensation process. The effects of poly(cyclohexanedimethylene terephthalate) (PCT) content on the copolymer are investigated by Fourier transform infrared spectroscopy (FT-IR), ${ }^{1} \mathrm{H}$ and ${ }^{13} \mathrm{C}$ nuclear magnetic resonance (NMR), gel permeation chromatographs (GPC), wide-angle X-ray diffraction (WAXD), differential scanning calorimetry (DSC), thermogravimetric analysis (TGA), mechanical, and visible light transmittance tests. FT-IR and NMR results confirm the incorporation of PCT onto the copolymer. WAXD and DSC indicate that the crystalline structure of the copolymers changed from $\alpha$-PBT lattice to trans-PCT lattice when the molar fraction of PCT $\left(M_{\mathrm{PCT}}\right)$ is above $30 \%$, while both crystallization and melting temperatures reach the minima. An increase in $M_{\mathrm{PCT}}$ led to an increase in the number sequence length of PCT, the thermal stability and the visible light transmittance of the copolymer, but to a slight decrease in tensile strength and elastic modulus.
\end{abstract}

Keywords: poly(ester ether); copolymerization; microstructures; crystallization behavior

\section{Introduction}

Thermoplastic poly(ether ester) elastomers (TPEEs) are usually block copolymers composed of polyester segment (hard segment) and polyether segment (soft segment) [1-3]. The hard segment forms physical crosslinks, while the soft segment is highly elastic and contributes to flexibility. Thus, in addition to possessing rubber-like properties, TPEEs can also be processed similar to thermoplastics. Because of the unique processing and mechanical properties, TPEEs are considered to be "third-generation synthetic rubbers", which are applied in numerous applications such as electronics and automotive industries, in skin tissue engineering, and so on [4-7].

Poly(butylene terephthalate) (PBT)-b-poly(tetramethylene glycol) (PTMG) is one of the most typical TPEEs with useful properties such as high tensile strength and elastic modulus. This is due to the thermodynamic immiscibility between the hard and soft segments, which dictates the crystallization behavior, morphology, and thermal and mechanical properties of PBT-b-PTMG $[8,9]$. 
Many attempts have been made to improve its properties. Celebi et al. attempted to add $\mathrm{TiO}_{2}$ or $\mathrm{ZnO}$ particles to the PBT-b-PTMG to improve its thermal stability, storage modulus, and mechanical properties [10]. Zhang et al. partially substituted aromatic segment PBT with aliphatic poly(butylene succinate) (PBS) to accelerate the degradation rate [11]. Liu et al. incorporated an aliphatic ring to partially replace the aromatic ring in PBT-b-PTMG to decrease its melting temperature without altering thermal stability [12].

However, the hard segment, PBT, is easy crystallized, which limits the transparency of TPEEs. Hence, there is still a need to improve properties of TPEEs with the emphasis on their transparency. The key to obtain this property is how to synchronize the mechanical properties with crystallization property such as degree of crystallinity, crystallization rate and crystallization temperature. Copolymerization is a powerful method to combine the advantages of corresponding homopolymers $[13,14]$. At the same time, it is also an effective way to decrease the average sequence length of the segments, as well as the degree of crystallinity [15].

It is worth noting poly(ethylene glycol-co-1,4-cyclohexanedimethanol terephthalate) (PETG) copolyester, which shows special crystallization behavior and high transparency [16-18]. PETG is prepared by partially replacing the ethylene glycol (EG) groups of poly(ethylene terephthalate) (PET) with 1,4-cyclohexanedimethanol (CHDM) groups. The PETG copolymer is essentially amorphous when CHDM content is in the range of $32-62 \%$, but its mechanical properties are close to those of PET [19]. CHDM has two isomers, trans (axial-axial or equatorial-equatorial) and cis (axial-equatorial), the former being more stable than the latter. Many investigations on the copolymers with cyclohexanedimethylene group reveal that the cyclohexane ring in the structure improves the thermal stability, mechanical properties and the transparency of the copolymer [20-24]. Therefore, the use of CHDM as a co-monomer makes the development of new types of optically interesting thermoplastic elastomers possible.

To improve the transparency of the copolymer, this work attempts to incorporate CHDM as a third co-monomer. The content of the hard segment PBT is decreased upon introducing PCT which has good crystallization behavior. The resulting copolymers are denoted as (PBT-co-PCT)-b-PTMG, which have constant soft segment length and molar fraction at $1000 \mathrm{~g} \mathrm{~mol}^{-1}$ and $20 \%$, respectively. The microstructure, crystallization behavior and properties of the copolymer are affected by the copolyester hard segments. Furthermore, compared to traditional TPEEs, the (PBT-co-PCT)-b-PTMG copolymers synthesized in this work show better performance in many aspects, such as high transparency and good thermal stability. For the sake of simplicity, they are designated by their theoretical PCT molar fractions $\left(M_{\mathrm{PCT}}\right)$ as PCT-M $M_{\mathrm{PCT}}$.

\section{Materials and Methods}

\subsection{Materials}

Dimethyl terephthalate (DMT), 1,4-butanediol (BDO), catalysts tetrabutyl titanate (TBT) and magnesium acetate $\left(\mathrm{MgAc}_{2}\right)$ were purchased from Sinopharm Chemical Reagent Co. Ltd. (Shanghai, China). CHDM was purchased from Aladdin Reagent Co. Ltd. (Shanghai, China); it was the mixture of $69 \%$ trans and $31 \%$ cis, and PTMG with an average molar weight of $1000 \mathrm{~g} \mathrm{~mol}^{-1}$ was supplied by BASF (Shanghai, China). The antioxidants (Irganox 1010 and Irgafos 168) were supplied by Ciba (Basel, Switzerland). All of these chemical materials were dried under vacuum at $80{ }^{\circ} \mathrm{C}$ for $24 \mathrm{~h}$ to a constant mass before use.

\subsection{Experiments}

(PBT-co-PCT)-b-PTMG copolymers were synthesized through molten polycondensation process in a dried steel reactor (Weihai Xingyu Chemical Machinery Co., Weihai, China) equipped with a stirrer, a condenser and a gas inlet. 
A typical procedure for the synthesis of (PBT-co-PCT)-b-PTMG copolymers is shown by the example of PCT50. The following chemicals were charged to the reactor under nitrogen: co-monomers DMT $(0.5 \mathrm{~mol})$, CHDM $(0.255 \mathrm{~mol}), \mathrm{BDO}(0.445 \mathrm{~mol})$ and PTMG $(100 \mathrm{~g})$; catalysts TBT $(0.1 \%$ of the total mass) and $\mathrm{MgAc}_{2}(0.02 \%$ of the total mass); and antioxidants Irganox 1010 and Irgafos 168 (0.5\% of the total mass). Then, the reactor was heated up to $205^{\circ} \mathrm{C}$ at $5{ }^{\circ} \mathrm{C} \mathrm{min}^{-1}$ and remained at that temperature for $3 \mathrm{~h}$ to reach the end of the transesterification. The polycondensation was then performed upon raising the temperature to $265^{\circ} \mathrm{C}$ and the pressure was below $50 \mathrm{~Pa}$. After $3.5 \mathrm{~h}$, the copolymer was extruded from the reactor under nitrogen, cooled down in a water bath, and dried in a vacuum dryer at $50{ }^{\circ} \mathrm{C}$ for $24 \mathrm{~h}$ to a constant mass.

It is worth noting that the reaction time and temperature were increased slightly with the increasing CHDM content. The reactions involved in these syntheses were formulated in Figure 1 and the compositions of all samples are given in Table 1.

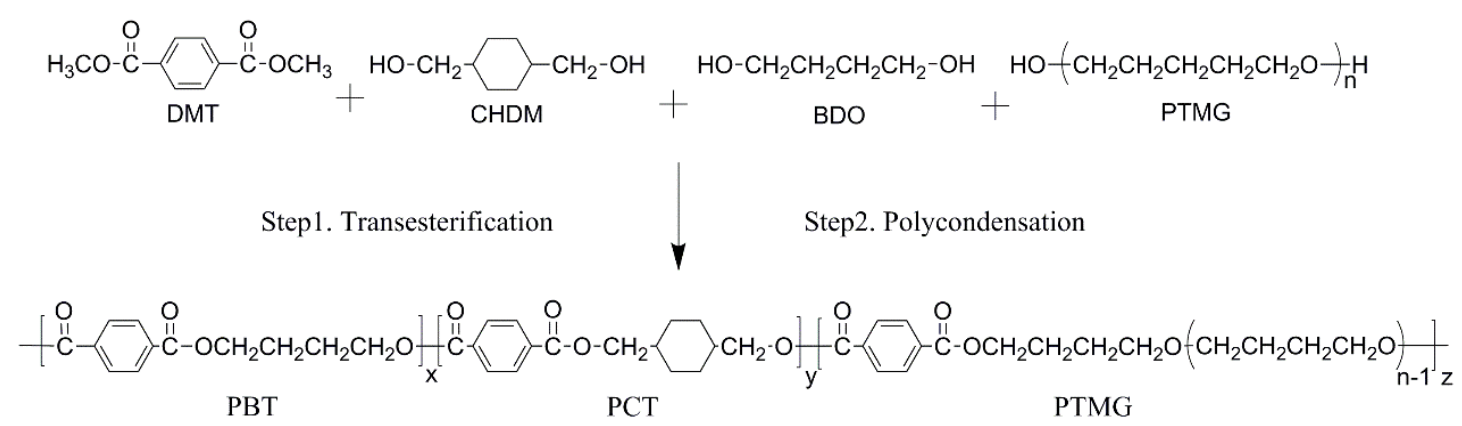

Figure 1. Synthesis route of the (PBT-co-PCT)-b-PTMG copolymers.

Table 1. Compositions for the synthesis of the (PBT-co-PCT)-b-PTMG copolymers.

\begin{tabular}{ccccc}
\hline Samples & DMT (mol) & CHDM (mol) & BDO (mol) & PTMG (g) \\
\hline PCT0 & 0.5 & - & 0.7 & 100 \\
PCT10 & 0.5 & 0.052 & 0.648 & 100 \\
PCT30 & 0.5 & 0.153 & 0.547 & 100 \\
PCT50 & 0.5 & 0.255 & 0.445 & 100 \\
PCT70 & 0.5 & 0.355 & 0.345 & 100 \\
\hline
\end{tabular}

\subsection{Characterization}

ATR-FTIR spectroscopy was performed on a FTIR spectrometer in the attenuated total reflectance mode (PE-Spectrum one, Perkinelmer, Waltham, MA, USA). The spectra were scanned from 600 to $4000 \mathrm{~cm}^{-1}$ with a resolution of $4 \mathrm{~cm}^{-1}$.

${ }^{1} \mathrm{H}$ NMR and ${ }^{13} \mathrm{C}$ NMR spectra were recorded on a spectrometer of Inova-600, USA, operating at $600 \mathrm{MHz}$. They were used for determining the copolymer composition and average sequence length, respectively. Deuterated chloroform $\left(\mathrm{CDCl}_{3}\right)$ was used as a solvent and tetramethylsilane (TMS) as an internal reference.

The number average molecular weight $\left(M_{\mathrm{n}}\right)$ and the dispersity $\left(M_{\mathrm{w}} / M_{\mathrm{n}}\right)$ were determined by GPC (PL-GPC220, Agilent Technologies, CA, USA). The GPC measurements were carried out at $150{ }^{\circ} \mathrm{C}$ using 1,2,4-trichlorobenzene as the eluent at a flow rate of $1 \mathrm{~mL} \mathrm{~min}^{-1}$.

Wide-angle X-ray diffraction patterns were recorded on a WAXD diffractometer of type Bruker D8 Advance (Bruker, Karlsruhe, Germany) in the $2 \theta$ range from $5^{\circ}$ to $40^{\circ}$ at a scanning rate of $2^{\circ} \mathrm{min}^{-1}$ for the copolymers prepared by a hot press. The crystal size in the perpendicular direction of the planes was calculated using the Scherrer's formula [25]:

$$
L=K \lambda / \beta \cos \theta
$$


where $L$ is the crystal size; $K$ is a structure constant, taken as $1 ; \lambda$ is the wavelength of the monochromatic $X$-ray beam $\left(0.154 \mathrm{~nm}\right.$ for $\mathrm{CuK}_{\alpha}$ radiation); $\beta$ is the full width at half maximum for the diffraction peak (rad); and the $\theta$ is the Bragg angle $\left(^{\circ}\right)$.

A differential scanning calorimeter of type Q-2000 (TA, Newcastle, PE, USA) was used to investigate the thermal properties of the copolymers. The measurement was performed using about $10 \mathrm{mg}$ sample sealed in an aluminum pan under the nitrogen atmosphere. Firstly, the sample was heated to $300{ }^{\circ} \mathrm{C}$ rapidly and kept isothermally for $1 \mathrm{~min}$ to remove the previous thermal history. Secondly, the sample was quenched to $-80{ }^{\circ} \mathrm{C}$ and heated to $300{ }^{\circ} \mathrm{C}$ at a speed of $10{ }^{\circ} \mathrm{C} \mathrm{min}{ }^{-1}$ to detect the glass transition temperature. Finally, a second cool scan and a third heat scan was performed at a speed of $10{ }^{\circ} \mathrm{C} \mathrm{min}^{-1}$ to investigate the thermal properties of the copolymers. The crystallinity of hard segments was measured from the heat fusion obtained from DSC curves using Equation (2) according to group contribution theory [26,27],

$$
W C=\frac{\Delta H_{m}}{m_{P B T} \times \Delta H_{P B T}^{0}+m_{P C T} \times \Delta H_{P C T}^{0}}
$$

where $\Delta H_{P B T}^{0}\left(144.5 \mathrm{~J} \mathrm{~g}^{-1}\right)$ [28] and $\Delta H_{P C T}^{0}\left(102.0 \mathrm{~J} \mathrm{~g}^{-1}\right)$ [29] are the heats of fusion of 1 mol PBT and PCT perfect crystals, respectively; and the mass fractions of the two types of hard segments are designated as $m_{P B T}$ and $m_{P C T}$, respectively.

The thermal stability of the copolymers was evaluated by TGA of type TASDT-Q600 (TA, Newcastle, PE, USA). The measurement was performed under nitrogen and oxygen flow, respectively. The heating rate was $20{ }^{\circ} \mathrm{C} \mathrm{min}^{-1}$ in range from 30 to $800{ }^{\circ} \mathrm{C}$.

The specimens for tensile testing were prepared by a hot press with the following dimensions: $33 \times 6.2 \times 2 \mathrm{~mm}^{3}$. Stress-strain curves were recorded by a CMT4104 testing machine (SUNS, Shenzhen, China) at a constant crosshead speed of $50 \mathrm{~mm} \mathrm{~min}^{-1}$, according to the standard ISO 37-2011. At least five specimens were tested and the average values of the elastic modulus $(E)$, tensile strength $\left(\sigma_{\max }\right)$ and elongation at break $\left(\varepsilon_{b}\right)$ were calculated.

The specimens for optical testing were prepared by a hot press and their thickness was $2 \mathrm{~mm}$. The visible light transmittance and haze were recorded on a Transmittance haze tester (WGT-S, Shanghai, China) according to ISO 14782.

\section{Results and Discussion}

\subsection{Structure and Molecular Weight Analysis}

The structure of those copolymers is first probed by a FTIR spectrometer in an ATR mode. Figure 2 shows the spectrum of PCT50 as an example. The peaks at 2941 and $2862 \mathrm{~cm}^{-1}$ are assigned to the asymmetric and symmetric aliphatic $\mathrm{C}-\mathrm{H}$ stretching vibrations of $\mathrm{PBT}[10]$. $\mathrm{C}=\mathrm{O}$ stretching peak and ester group skeleton peak are observed at 1715 and $1268 \mathrm{~cm}^{-1}$, respectively. The $\mathrm{C}-\mathrm{O}-\mathrm{C}$ skeleton asymmetric vibration of soft segment, $\mathrm{CH}_{2}$ bending vibration, $\mathrm{C}-\mathrm{H}$ stretching vibration of cyclohexylene ring and $\mathrm{C}-\mathrm{H}$ vibration of benzene ring appear at 1104, 1454, 958 and $727 \mathrm{~cm}^{-1}$, respectively [30].

Figure 3 shows a typical ${ }^{1} \mathrm{H}$ NMR spectrum of PCT50, which corroborates its ATR-FTIR spectrum. Peak a at $8.01 \mathrm{ppm}$ is the characteristic peak of protons in the benzene ring and stands for the total molar content of the acidic moieties herein. Peaks b and c, with chemical shifts at 4.35 and 1.98 ppm, respectively, correspond to the methylene protons of the hard segments PBT. The splits with chemical shift at 4.21 and $4.11 \mathrm{ppm}$ are ascribed to the resonance peaks of methylene protons in CHDM moieties with cis and trans conformation and marked as $d_{c}$ and $d_{t}$, respectively. The proton resonances of the cyclohexylene group appear between $1.08 \mathrm{ppm}$ and $1.86 \mathrm{ppm}$ and are marked as $\mathrm{e}_{1}, \mathrm{e}_{2}, \mathrm{e}_{3}, \mathrm{e}_{4}$ and $\mathrm{e}_{5}$, respectively. Peak f at $3.40 \mathrm{ppm}$ and $\mathrm{g}$ at $1.53 \mathrm{ppm}$ correspond to the soft segment. The chemical shifts of the tetramethylene protons attached to the ester group of the soft segment are peaks $\mathrm{f}_{1}$ at $4.36 \mathrm{ppm}$ and $\mathrm{g}_{1}$ at 1.59 ppm [31,32]. 


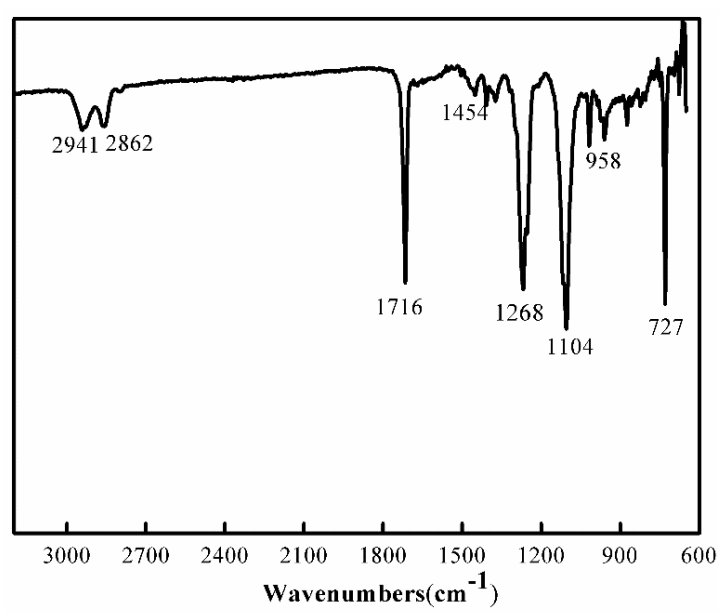

Figure 2. ATR-FTIR spectra of the sample PCT50.
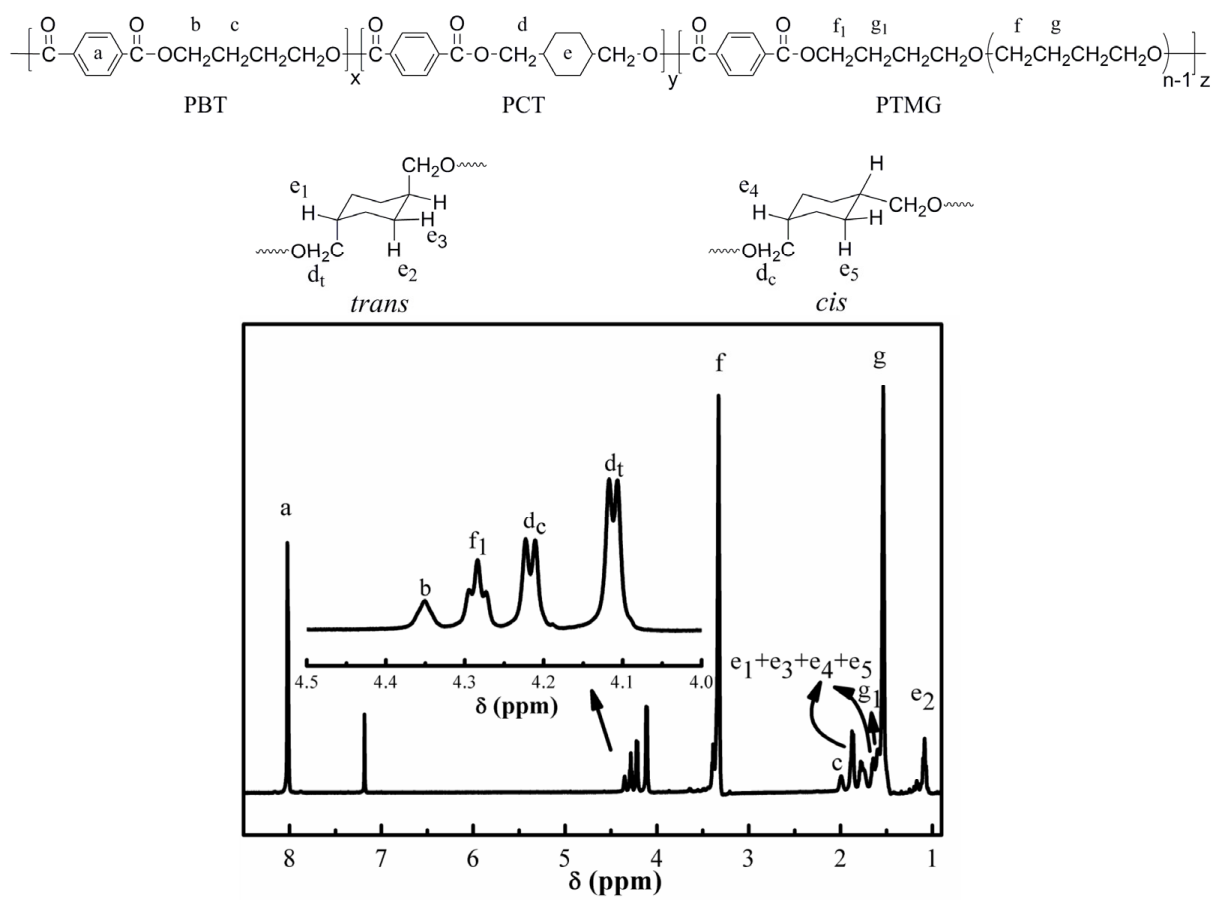

Figure 3. ${ }^{1} \mathrm{H}$ nuclear magnetic resonance spectra of sample PCT50.

Based on the ${ }^{1} \mathrm{H}$ NMR spectra, the copolymer compositions can be determined from the respective peak areas as follows:

$$
\begin{gathered}
M_{P B T}=\frac{I_{b}}{I_{a}} \\
M_{P C T}=\frac{I_{d_{c}}+I_{d_{t}}}{I_{a}} \\
M_{P T M G}=\frac{I_{f_{1}}}{I_{a}}
\end{gathered}
$$

where $M_{\mathrm{PBT}}, M_{\mathrm{PCT}}$ and $M_{\mathrm{PTMG}}$ denote the molar fractions of butylene terephthalate (BT), 1,4-cyclohexanedimethanol terephthalate (CT) and PTMG, respectively. $I_{a}, I_{b}, I_{d c}, I_{d t}$ and $I_{f 1}$ represent the integral areas of peaks $a, b, d_{c}, d_{t}$ and $f_{1}$ in Figure 3 , respectively. Table 2 shows that the compositions calculated by Equations (3)-(5) match relatively well the feed compositions. 
Table 2. Compositions, molecular weights and polydispersity of the (PBT-co-PCT)-b-PTMG copolymers.

\begin{tabular}{cccccccc}
\hline Samples & $\boldsymbol{M}_{\boldsymbol{P T M G}} \mathbf{( \% )}$ & $\boldsymbol{M}_{\boldsymbol{P B T}} \mathbf{( \% )}$ & $\boldsymbol{M}_{\boldsymbol{P C T}} \mathbf{( \% )}$ & trans $/$ cis & $\boldsymbol{M}_{\mathbf{n}}$ & $\boldsymbol{M}_{\mathbf{w}}$ & PDI \\
\hline PCT0 & 20.2 & 79.1 & - & - & $1.8 \times 10^{4}$ & $3.7 \times 10^{4}$ & 2.08 \\
PCT10 & 19.8 & 69.6 & 10.6 & $69.8 / 30.2$ & $1.7 \times 10^{4}$ & $3.6 \times 10^{4}$ & 2.12 \\
PCT30 & 19.7 & 49.5 & 30.8 & $70.3 / 29.7$ & $1.7 \times 10^{4}$ & $3.5 \times 10^{4}$ & 2.11 \\
PCT50 & 20.1 & 28 & 51.9 & $71.6 / 28.4$ & $1.6 \times 10^{4}$ & $3.3 \times 10^{4}$ & 2.07 \\
PCT70 & 20.3 & 7.6 & 72.1 & $69.1 / 30.9$ & $1.5 \times 10^{4}$ & $3.1 \times 10^{4}$ & 2.06 \\
\hline
\end{tabular}

The theoretical $M_{\text {PTMG }}$ is fixed at $20 \%$ and the measured values are very close to it. However, the measured values of $M_{\mathrm{PCT}}$ are always slightly higher than the theoretical ones. This is because CHDM is more difficult to remove than BDO during the polycondensation under the vacuum. Moreover, BDO participates in side reactions during the transesterification upon forming tetrahydrofuran. It is also interesting to note that the molar ratio of trans to cis isomers of the CT is almost constant at about 2.3 to 1 for all copolymers. This indicates that isomerization between trans and cis of CHDM did not occur during the polymerization.

Table 2 also shows the molecular weights of the copolymers. Both the number and weight average molecular weights of the copolymers decrease very slightly with increasing CT content. This is because it is more difficult to remove CHDM than BDO during the polycondensation under the vacuum.

\subsection{Sequence Distribution Analysis}

Since Yamadera and Murano [33] proposed a method to determine the average sequence length and the distribution of copolyesters, many studies focused on the analysis of the microstructures of copolymers by ${ }^{13} \mathrm{C}$ NMR [34-36]. Figure 4 shows the ${ }^{13} \mathrm{C}$ NMR spectra of the (PBT-co-PCT)-b-PTMG copolymers. The quaternary carbon atom resonance in benzene rings splits into five peaks. Peak $b$ is due to the quaternary carbon atom of the benzene ring linked to the soft segment PTMG because its relative integral intensity changes slightly with increasing $M_{\mathrm{PCT}}$ at a constant molar fraction of soft segment. The sequence distribution of the two hard segments, PBT and PCT, is analyzed according to the following procedure.

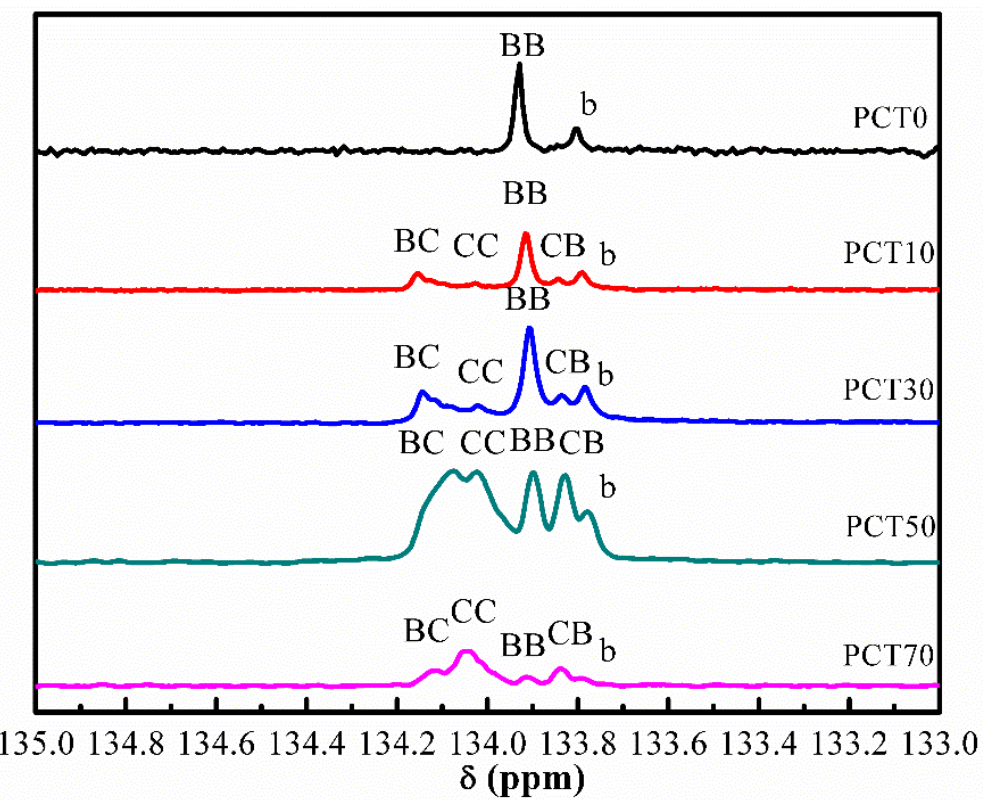

Figure 4. ${ }^{13} \mathrm{C}$ NMR spectra of the (PBT-co-PCT)-b-PTMG copolymers. 
The molar fractions of $B T$ moiety and $C T$ moiety are determined from integration of the peaks. $P_{B}$ $\left(P_{C}\right)$ is the molar fraction of the $B T(C T)$ moiety, and $f_{B B}, f_{B C}, f_{C B}$ and $f_{C C}$ correspond to the proportions of the integrated intensities of four types of dyads, $B B, B C, C B$ and $C C$, respectively. Then, $P_{B C}\left(P_{C B}\right)$ is the probability of finding a $B T(C T)$ moiety next to a $C T(B T)$ moiety. The number average sequence lengths of $B T$ and CT moieties are designed by $L_{n B}$ and $L_{n C}$, respectively. The degree of randomness is designated by $D R$ and is equal to 0 for a mixture of homopolymers, and is equal to 2 for an alternating distribution $[37,38]$. The above parameters can be calculated by the following equations:

$$
\begin{gathered}
P_{B}=\frac{f_{B C}+f_{C B}}{2}+f_{B B} P_{C}=\frac{f_{B C}+f_{C B}}{2}+f_{C C} \\
P_{B C}=\frac{f_{B C}+f_{C B}}{2 P_{B}} P_{C B}=\frac{f_{B C}+f_{C B}}{2 P_{C}} \\
L_{n B}=\frac{2 P_{B}}{f_{B C}+f_{C B}} L_{n C}=\frac{2 P_{C}}{f_{B C}+f_{C B}} \\
D R=p_{B C}+P_{C B}
\end{gathered}
$$

Their values are gathered in Table 3. All the values of $D R$ are very close to 1 , indicating that the sequence distribution in the copolymers is random in the entire range of the CT composition. The number average sequence length $L_{n B}$ decreases while $L_{n} C$ increases with increasing CT content, as expected. This is due to the increasing content of monomer CHDM, which leads to an increase of the probability that the bis(hydroxymethylcyclohexane)terephthalate (BHCT) reacts with BHCT during the polycondensation reaction.

Table 3. Sequence distribution analysis of the (PBT-co-PCT)-b-PTMG copolymers.

\begin{tabular}{ccccccccc}
\hline \multirow{2}{*}{ Samples } & \multicolumn{3}{c}{ Fraction of Dyad } & \multicolumn{2}{c}{$\begin{array}{c}\text { Molar Fraction of } \\
\text { Diester }\end{array}$} & $\begin{array}{c}\text { Average } \\
\text { Sequence Length }\end{array}$ & $\begin{array}{c}\text { Degree of } \\
\text { Randomness }\end{array}$ \\
\cline { 2 - 9 } & $f_{B B}$ & $f_{B C}+f_{C B}$ & $f_{C C}$ & $\boldsymbol{P}_{\boldsymbol{B}}$ & $\boldsymbol{P}_{\boldsymbol{C}}$ & $\boldsymbol{L}_{\boldsymbol{n} \boldsymbol{B}}$ & $\boldsymbol{L}_{\boldsymbol{n}}$ & $\boldsymbol{D R}$ \\
\hline PCT0 & - & - & - & - & - & - & - & - \\
PCT10 & 0.578 & 0.382 & 0.04 & 0.778 & 0.222 & 4.1 & 1.2 & 1.05 \\
PCT30 & 0.456 & 0.441 & 0.103 & 0.677 & 0.323 & 3.1 & 1.5 & 1.01 \\
PCT50 & 0.192 & 0.483 & 0.325 & 0.434 & 0.566 & 1.8 & 2.3 & 0.98 \\
PCT70 & 0.112 & 0.462 & 0.426 & 0.343 & 0.657 & 1.5 & 2.8 & 1.02 \\
\hline
\end{tabular}

\subsection{WAXD Analysis}

Figure 5 shows the WAXD diagrams of the (PBT-co-PCT)-b-PTMG copolymers. The intensities of the peaks increase with the increasing PCT content. Table 4 shows the corresponding WAXD data.

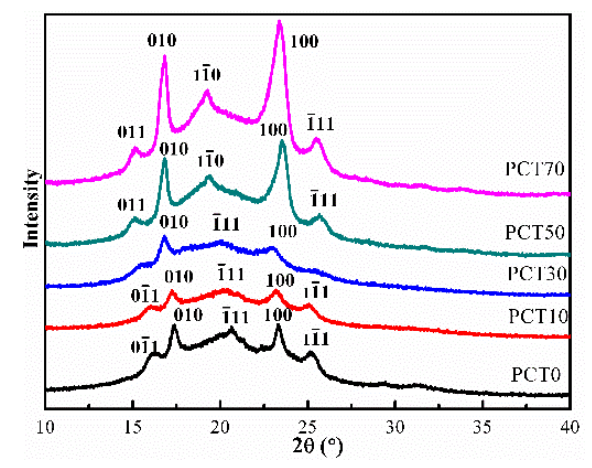

Figure 5. WAXD patterns of the (PBT-co-PCT)-b-PTMG copolymers. 
Table 4. WAXD data of the (PBT-co-PCT)-b-PTMG copolymers.

\begin{tabular}{|c|c|c|c|c|c|}
\hline Samples & Crystal Plane & $2 \theta\left({ }^{\circ}\right)$ & $d(\AA ̊)$ & $\beta\left({ }^{\circ}\right)$ & $L(\AA)$ \\
\hline \multirow{5}{*}{ РCT0 } & $0 \overline{1} 1$ & 16.27 & 5.46 & 0.59 & 151.14 \\
\hline & 010 & 17.39 & 5.10 & 0.43 & 207.69 \\
\hline & $\overline{1} 11$ & 20.65 & 4.29 & 0.96 & 93.47 \\
\hline & 100 & 23.33 & 3.80 & 0.61 & 147.77 \\
\hline & $1 \overline{1} 1$ & 25.20 & 3.52 & 0.64 & 141.34 \\
\hline \multirow{5}{*}{ PCT10 } & $0 \overline{1} 1$ & 16.06 & 5.51 & 0.61 & 146.15 \\
\hline & 010 & 17.25 & 5.13 & 0.45 & 198.42 \\
\hline & $\overline{1} 11$ & 20.36 & 4.35 & 0.98 & 91.52 \\
\hline & 100 & 23.19 & 3.83 & 0.67 & 134.50 \\
\hline & $1 \overline{1} 1$ & 25.07 & 3.54 & 0.66 & 137.02 \\
\hline \multirow{5}{*}{ РСТ30 } & - & - & - & - & - \\
\hline & 010 & 16.80 & 5.27 & 0.47 & 189.87 \\
\hline & $\overline{1} 11$ & 20.16 & 4.40 & 1.01 & 88.78 \\
\hline & 100 & 22.96 & 3.87 & 0.82 & 109.8 \\
\hline & - & - & - & - & - \\
\hline \multirow{5}{*}{ РCT50 } & 011 & 15.13 & 5.85 & 0.53 & 168.02 \\
\hline & 010 & 16.83 & 5.27 & 0.46 & 193.99 \\
\hline & $1 \overline{1} 0$ & 19.36 & 4.58 & 0.77 & 116.31 \\
\hline & 100 & 23.56 & 3.77 & 0.66 & 136.64 \\
\hline & $\overline{1} 11$ & 25.72 & 3.46 & 0.66 & 137.20 \\
\hline \multirow{5}{*}{ PCT70 } & 011 & 15.17 & 5.83 & 0.47 & 189.49 \\
\hline & 010 & 16.83 & 5.26 & 0.45 & 198.31 \\
\hline & $1 \overline{1} 0$ & 19.24 & 4.61 & 0.73 & 122.66 \\
\hline & 100 & 23.39 & 3.79 & 0.64 & 140.86 \\
\hline & $\overline{1} 11$ & 25.50 & 3.49 & 0.65 & 139.24 \\
\hline
\end{tabular}

According to Briber and Thomas [39], hard segments PBT moieties in a thermoplastic elastomer always organize in the $\alpha$-PBT form when crystallized from the molten state, and the lattice is independent of the concentration of soft segment. Therefore, for the (PBT-co-PCT)-b-PTMG copolymers when $M_{\mathrm{PCT}}$ is below $30 \%$, the hard segments are organized in the form of $\alpha$-PBT crystal. The peaks (and the corresponding Miller indices) of the $\alpha$-PBT at $2 \theta$ are $16.12^{\circ}(0 \overline{1} 1), 17.38^{\circ}(010), 20.91^{\circ}(\overline{101})$, $23.36^{\circ}(100)$, and $25.31^{\circ}(1 \overline{1} 1)$. The unit cell of $\alpha$-PBT is triclinic with the cell parameters $a=0.483 \mathrm{~nm}$, $b=0.594 \mathrm{~nm}, c=1.159 \mathrm{~nm}$ and $\alpha=99.7^{\circ}, \beta=115.2^{\circ}$, and $\gamma=110.8^{\circ}$, according to the reported literature [40].

Boye [41] reported that WAXD pattern of PCT homopolymer with a trans/cis isomer ratio of more than 68/32 was the same as the pure trans-PCT. In this study, trans/cis isomer ratio is around to 70/30, as shown in Table 1. Since the trans/cis isomer ratios of the (PBT-co-PCT)-b-PTMG copolymers are about 70/30, their CT moieties for $M_{\mathrm{PCT}}$ of more than $30 \%$ are expected to form triclinic crystal lattices with the reported cell parameters: $a=0.637 \mathrm{~nm}, b=0.663 \mathrm{~nm}$, and $c=1.42 \mathrm{~nm}$; and $\alpha=89.35^{\circ}$, $\beta=47.11^{\circ}$, and $\gamma=134.36^{\circ}$. The peaks (and the corresponding Miller indices of trans-PCT) at $2 \theta$ are $14.94^{\circ}(011), 16.68^{\circ}(1 \overline{1} 1), 19.32^{\circ}(1 \overline{1} 0), 23.26^{\circ}(100)$, and $25.57^{\circ}(\overline{1} 11)$, according to the reported literature [32].

Figure 5 shows that when $M_{\mathrm{PCT}}$ is less than $30 \%$, the CT moieties do not intervene in the formation of $\alpha$-PBT crystal lattices. When it is above 30\%, the intensities of the peaks corresponding to the trans-PCT crystal lattices increase rapidly with increasing $M_{\mathrm{PCT}}$ because CT moieties with an average sequence length exceeding 1.5 can crystallize. The transition from $\alpha$-PBT crystal lattices to trans-PCT crystal lattices of the (PBT-co-PCT)-b-PTMG copolymers can be explained from the viewpoint of cohesive energy (E).

Cohesive energy (E) is used to describe the energetic interactions between the segments of each component polymer molecules in the molten state. The cohesive energies corresponding to A and $\mathrm{B}$ 
components in an A/B binary random copolymer are assumed to be proportional to the copolymer composition and can be calculated according to the group contribution method [29,31]. When the cohesive energies of A and B components are identical, both A and B can co-crystallize into a crystal lattice at certain compositions. According to the group contribution method, the cohesive energies of hard segments PBT and PCT are $89,420 \mathrm{~J} \mathrm{~mol}^{-1}$ and 119,160 $\mathrm{J} \mathrm{mol}^{-1}$ [15], respectively. Moreover, the PCT composition of the (PBT-co-PCT)-b-PTMG copolymer at which the two hard segments PBT and PCT undergo co-crystallization, denoted as $M_{\mathrm{PCT}}^{*}$, is $43 \%$. This value is close to the molar fraction of the CT hard segments in Table 1 . That is to say, theoretically, when $M_{\mathrm{PCT}}$ is less than $M_{\mathrm{PCT}}^{*}$, only BT moieties can be crystallize and form $\alpha$-PBT crystal lattices, and the CT moieties would be repelled into non-crystallized regions; and, when $M_{\mathrm{PCT}}$ is above $M_{\mathrm{PCT}}^{*}, \mathrm{CT}$ moieties begin to crystallize and form trans-PCT crystal lattices, while the BT moieties would be repelled into non-crystallized regions. It is worth noting that the crystal lattice transition from $\alpha$-PBT to trans-PCT also manifests itself by the minimum melting temperature, minimum heat of fusion, and the discontinuous jump of the glass transition temperature for the (PBT-co-PCT)-b-PTMG copolymer, as will be shown below.

In Table 4, the crystal size of the $\alpha$-PBT crystals of PCT0 are larger than those of PCT10 and PCT30 under the same crystallization condition, and those of the trans-PCT crystals of PCT70 are larger than those of PCT50. These results indicate that the crystal size of the (PBT-co-PCT)-b-PTMG copolymer decreases first and then increases with the increasing CT content.

\subsection{DSC Analysis}

Figure 6 shows the DSC traces of the (PBT-co-PCT)-b-PTMG copolymers and Table 5 gathers the values of the glass transition temperatures of the soft and hard segments $\left(T_{g s}\right.$ and $\left.T_{g h}\right)$, crystallization temperature $\left(T_{\mathcal{c}}\right)$, melting temperature $\left(T_{m}\right)$ and enthalpies of crystallization $\left(\Delta H_{\mathcal{c}}\right)$ and fusion $\left(\Delta H_{m}\right)$. In Figure 6a, all copolymers crystallize when cooled down from the molten state as they all exhibit a crystallization peak. The crystallization temperature decreases with the increasing CT content until 30\%; and it increases with the increasing CT content when exceeding 30\%. The enthalpy of crystallization follows the same trend and reaches a minimum at $M_{\mathrm{PCT}}$ of around $30 \%$. At around this CT content, none of the average sequences of the PBT and PCT hard segments is long enough to fully crystallize.

From the DSC heating traces (Figure 6b), the melting temperature decreases first from $187.8^{\circ} \mathrm{C}$ to $150.6{ }^{\circ} \mathrm{C}$ and then increases again to $245.2{ }^{\circ} \mathrm{C}$. Meanwhile, the enthalpy of fusion $\left(\Delta H_{m}\right)$ and the degree of crystallinity $\left(X_{C}\right)$ follow a trend similar to that of the melting temperature, and reach minima when the $C T$ content is around $30 \%$, for the reason above.

Table 5. Thermal properties of the (PBT-co-PCT)-b-PTMG copolymers obtained from DSC.

\begin{tabular}{cccccccc}
\hline \multirow{2}{*}{ Samples } & \multicolumn{5}{c}{ Cooling } & \multicolumn{5}{c}{ Heating } \\
\cline { 2 - 7 } & $\boldsymbol{T}_{\boldsymbol{c}}\left({ }^{\circ} \mathrm{C}\right)$ & $\boldsymbol{\Delta} \boldsymbol{H}_{\boldsymbol{c}} \mathbf{( J \cdot \mathbf { g } ^ { - \mathbf { 1 } } )}$ & $\boldsymbol{T}_{\boldsymbol{g} s}\left({ }^{\circ} \mathrm{C}\right)$ & $\boldsymbol{T}_{\boldsymbol{g h}}\left({ }^{\circ} \mathrm{C}\right)$ & $\boldsymbol{T}_{\boldsymbol{m}}\left({ }^{\circ} \mathrm{C}\right)$ & $\boldsymbol{\Delta} \boldsymbol{H}_{\boldsymbol{m}}\left(\mathbf{J} \cdot \mathbf{g}^{-\mathbf{1}}\right)$ & $\boldsymbol{W}_{\boldsymbol{C}}(\mathbf{\%})$ \\
\hline PCT0 & 147.6 & 22.6 & -16.1 & 33.8 & 187.8 & 22.8 & 19.9 \\
PCT10 & 118.1 & 17.0 & -16.7 & 26.4 & 158.8 & 17.7 & 15.9 \\
PCT30 & 83.9 & 12.0 & -17.0 & 23.5 & 150.6 & 12.1 & 10.5 \\
PCT50 & 147.9 & 13.7 & -11.9 & 42.9 & 206.5 & 14.0 & 15.0 \\
PCT70 & 198.0 & 15.6 & -12.7 & 45.5 & 245.2 & 15.7 & 18.6 \\
\hline
\end{tabular}




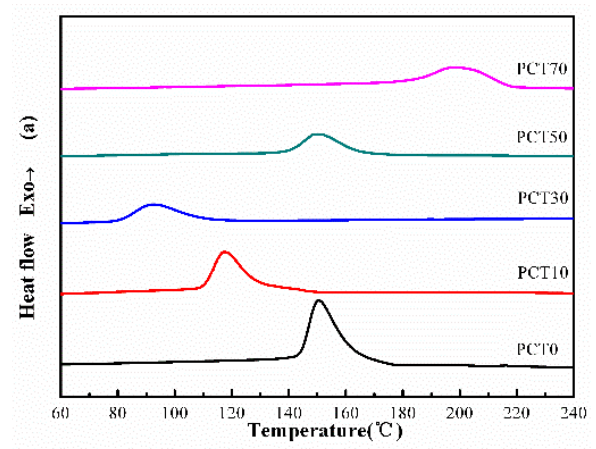

(a)

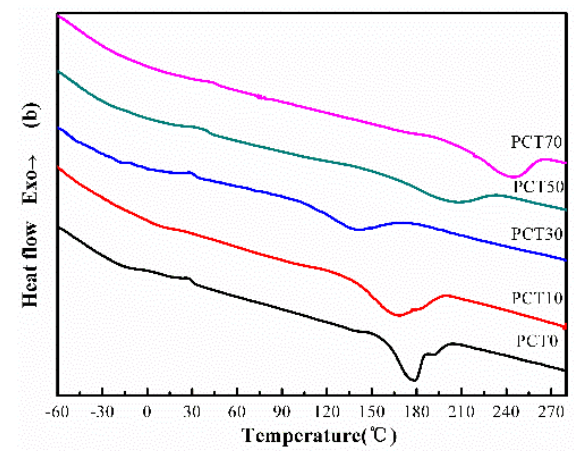

(b)

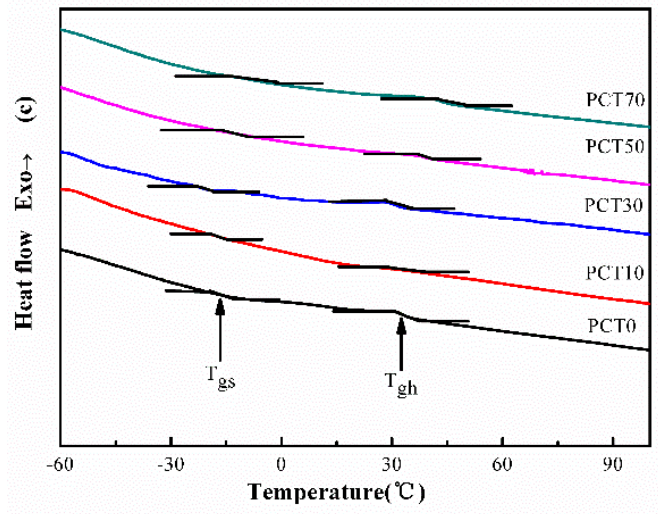

(c)

Figure 6. DSC traces of the (PBT-co-PCT)-b-PTMG copolymers at $10{ }^{\circ} \mathrm{C} \mathrm{min}^{-1}$ in nitrogen: (a) cooling traces; (b) heating traces; and (c) heating traces of the quenched samples.

Furthermore, Figure $6 \mathrm{c}$ shows the glass transition temperature of the (PBT-co-PCT)-b-PTMG copolymers previously quenched from the melt. Their value is estimated through the tangent midpoint of the curves. The glass transition temperature of the soft segments PTMG $\left(T_{g_{s}}\right)$ appears in the vicinity of $-14{ }^{\circ} \mathrm{C}$, which is slightly higher than that of PTMG homopolymer because of the loss of free volume of the amorphous domains with the interference of the amorphous hard segments. On the other hand, the glass transition temperature of the hard segments PBT and PCT $\left(T_{g h}\right)$ decreased with increasing CT content until $30 \%$. It is $33.8{ }^{\circ} \mathrm{C}$ for PCT0 and $23.5^{\circ} \mathrm{C}$ for PCT30. This is due to the structure regularity of the hard segments. When the CT content is less than $30 \%, T_{g h}$ is lower than that of PBT homopolymer because of the influence of the CT content in amorphous. Thereafter, when the CT content is above $30 \%$, the structure of hard segments become regular again, $T_{g h}$ increases with increasing CT content and reaches $45.5^{\circ} \mathrm{C}$ for PCT70.

\subsection{TGA Analysis}

Figure 7 shows the thermal gravimetrical traces of the (PBT-co-PCT)-b-PTMG copolymers. In Figure $7 \mathrm{a}, \mathrm{c}$, the thermal decomposition temperature increases with increasing $\mathrm{CT}$ content under nitrogen atmosphere, indicating that the thermal stability of the copolymer is improved by CT moieties. This is due to the increase of thermally stable cyclohexylene group [15]. 


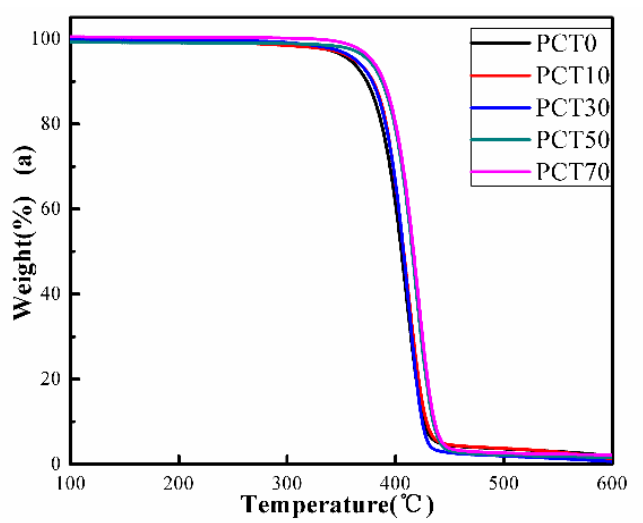

(a)

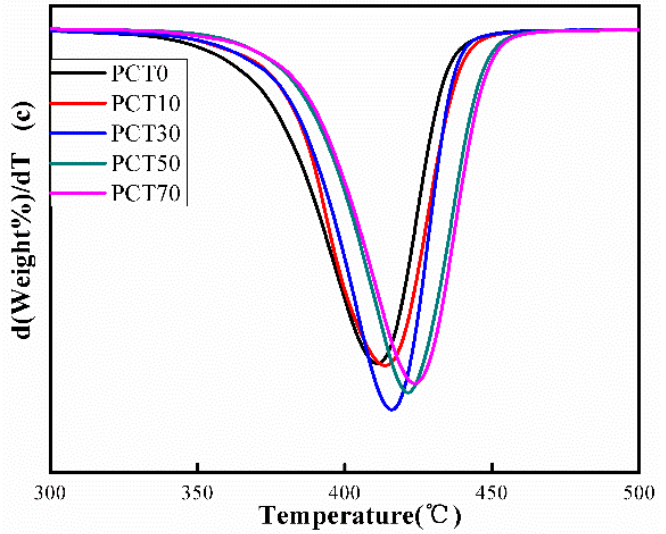

(c)

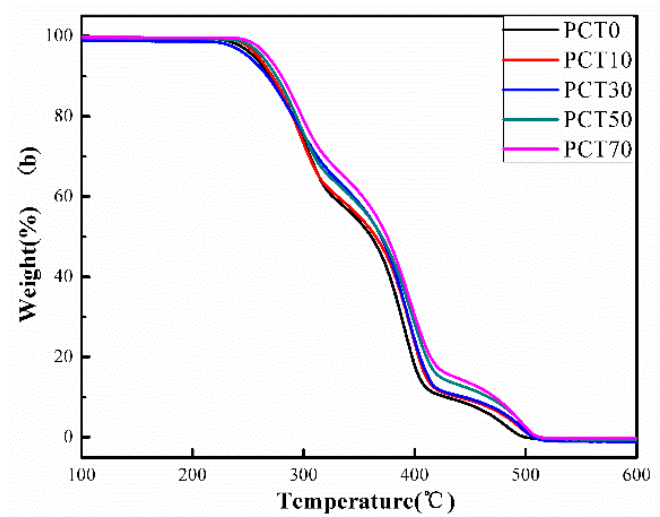

(b)

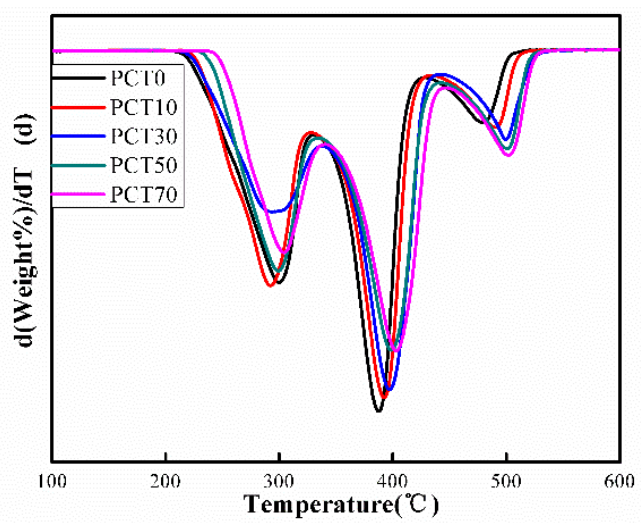

(d)

Figure 7. TGA and DTG thermograms of the (PBT-co-PCT)-b-PTMG copolymers at $20^{\circ} \mathrm{C} \mathrm{min}^{-1}$ : (a) TGA in nitrogen atmosphere; (b) TGA in oxygen atmosphere; (c) DTG in nitrogen atmosphere; and (d) DTG in oxygen atmosphere.

Compared with the thermal decomposition under nitrogen atmosphere, three degradation platforms are observed under the oxygen atmosphere in Figure $7 \mathrm{~b}, \mathrm{~d}$. The first and second platforms are assigned to the oxygen degradation of the ether bond in the soft segment and the copolyester in the hard segments, respectively [42,43]. It is worth noting that the CT moieties mainly increase the thermal stability of the hard segments of the copolymers. The third platform is due to the further oxidation of the oxidation products of the first and second stages.

\subsection{Mechanical Properties}

Figure 8 shows the stress-strain curves of the (PBT-co-PCT)-b-PTMG copolymers. Each curve is the average of five specimens. They all exhibit a typical thermoplastic elastomer behavior: an elastic deformation with small elongations, followed by a decrease in the slope without an apparent yield point. Moreover, the mechanical properties of a (PBT-co-PCT)-b-PTMG copolymer depends very much on its composition, especially the crystallinity of its hard segments. The elastic modulus (E) and tensile strength $\left(\sigma_{b}\right)$ of PCT30 are the lowest while its elongation at break is the highest, because its crystallinity is the lowest. Table 6 gathers the values of these properties for the (PBT-co-PCT)-b-PTMG copolymers synthesized in this work. 


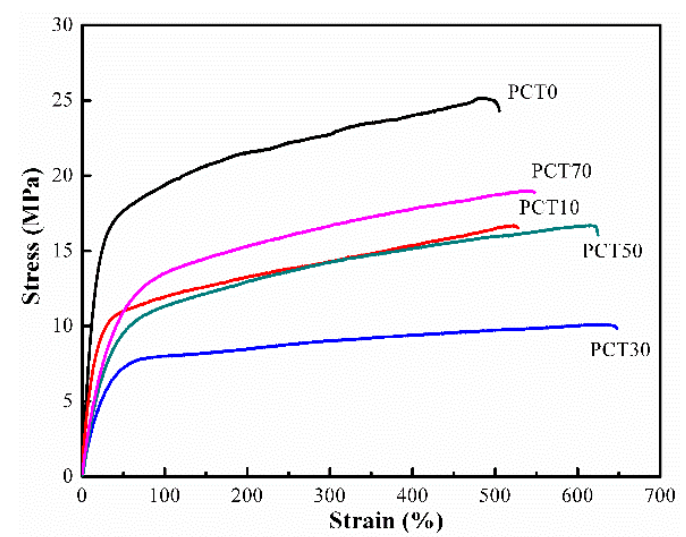

Figure 8. Tensile stress-strain curves of the (PBT-co-PCT)-b-PTMG copolymer.

Table 6. The mechanical properties for the (PBT-co-PCT)-b-PTMG copolymers

\begin{tabular}{cccc}
\hline Samples & Elastic Modulus (E) & Tensile Strength $\boldsymbol{\sigma}_{\max } \mathbf{( M P a )}$ & Elongation at Break $\varepsilon_{\mathbf{b}}(\mathbf{\%})$ \\
\hline PCT0 & $101.7( \pm 9.8)$ & $25.1( \pm 3.7)$ & $505.3( \pm 49.8)$ \\
PCT10 & $71.2( \pm 7.4)$ & $16.6( \pm 1.8)$ & $527.7( \pm 47.2)$ \\
PCT30 & $33.2( \pm 3.6)$ & $10.1( \pm 1.2)$ & $647.7( \pm 61.3)$ \\
PCT50 & $35.2( \pm 3.3)$ & $16.7( \pm 1.7)$ & $624.4( \pm 63.7)$ \\
PCT70 & $43.4( \pm 4.7)$ & $19.0( \pm 2.1)$ & $547.9( \pm 50.7)$ \\
\hline
\end{tabular}

\subsection{Optical Properties}

Figure 9 shows the visible light transmittance and haze of the (PBT-co-PCT)-b-PTMG copolymers as a function of their CT content. Overall, the visible light transmittance increases first and then levels off with the increasing CT content, and concomitantly the haze decreases first and then levels off with the increasing CT content. In other words, the incorporation of CT moieties improves the optical properties of the (PBT-co-PCT)-b-PTMG copolymer.

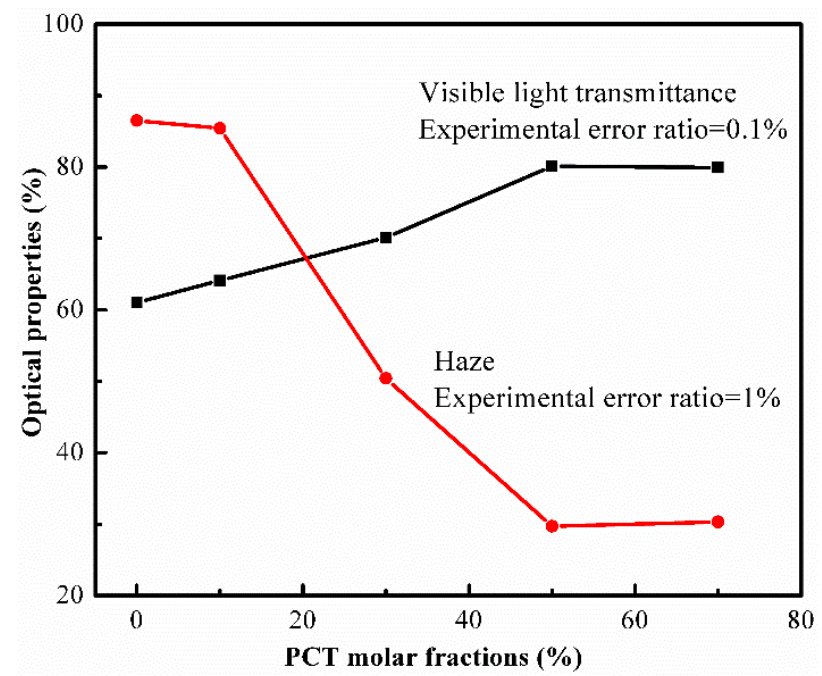

Figure 9. The optical properties of the (PBT-co-PCT)-b-PTMG copolymers.

\section{Conclusions}

In summary, this work shows that partial substitution of the BT hard segments of PBT-b-PTMG copolymers by CT moieties decreases the average sequence length of BT moiety and the crystallinity of the copolymers, resulting in a decrease in elastic modulus and tensile strength, and an increase in 
the average sequence length of CT moiety and elongation at break. On the other hand, it improves their thermal stability and optical properties.

Acknowledgments: This research did not receive any specific grant from funding agencies in the public, commercial, or not-for-profit sectors.

Author Contributions: This research was done by Yi-Cheng Feng and Hui Zhao, Tao Jiang and Tong-Hui Hao guided it, Yi-Cheng Feng finished this paper with the help of Qun-Chao Zhang, and it was revised by Guo-Hua Hu.

Conflicts of Interest: The authors declare no conflict of interest.

\section{References}

1. Orimo, Y.; Hotta, A. Stress-strain behavior, elastic recovery, fracture points, and time-temperature superposition of an oot-possessing triblock copolymer. Macromolecules 2011, 44, 5310-5317. [CrossRef]

2. Tomita, S.; Wataoka, I.; Igarashi, N.; Shimizu, N.; Takagi, H.; Sasaki, S.; Sakurai, S. Strain-induced deformation of glassy spherical microdomains in elastomeric triblock copolymer films: Time-resolved 2D-SAXS measurements under stretched state. Macromolecules 2017, 50, 677-686. [CrossRef]

3. Paszkiewicz, S.; Piesowicz, E.; Szymczyk, A. Phase separation and elastic properties of poly(trimethylene terephthalate)-block-poly(ethylene oxide) copolymers. Polymers 2016, 8, 237. [CrossRef]

4. Zhang, Z.Y.; Zhang, Q.K.; Yu, J.P.; Wu, Y.X.; Shen, Z.; Fan, X.H. Synthesis and properties of a new high-temperature liquid crystalline thermoplastic elastomer based on mesogen-jacketed liquid crystalline polymer. Polymer 2017, 108, 50-57. [CrossRef]

5. Zhang, K.; Gao, L.; Chen, Y. Organic/inorganic nanoobjects with controlled shapes from gelable triblock copolymers. Polymer 2010, 51, 2809-2817. [CrossRef]

6. Eberl, A.; Heumann, S.; Kotek, R.F.; Mitsche, S. Enzymatic hydrolysis of ptt polymers and oligomers. J. Biotechnol. 2008, 135, 45-51. [CrossRef] [PubMed]

7. Gao, L.; Zhang, K.; Chen, Y. Functionalization of shaped polymeric nanoobjects via bulk co-self-assembling gelable block copolymers with silane coupling agents. Polymer 2011, 52, 3681-3686. [CrossRef]

8. Gabriëlse, W.; Maria Soliman, A.; Dijkstra, K. Microstructure and phase behavior of block copoly(ether ester) thermoplastic elastomers. Macromolecules 2001, 34, 1685-1693. [CrossRef]

9. Szymczyk, A. Structure and properties of new polyester elastomers composed of poly(trimethylene terephthalate) and poly(ethylene oxide). Eur. Polym. J. 2009, 45, 2653-2664. [CrossRef]

10. Celebi, H.; Bayram, G.; Dogan, A. Effect of zno type and concentration on the mophology, thermal, and mechanical properties of poly(ether ester)/ZnO composites. J. Appl. Polym. Sci. 2013, 129, 3417-3424. [CrossRef]

11. Szymczyk, A.; Nastalczyk, J.; Sablong, R.J.; Roslaniec, Z. The influence of soft segment length on structure and properties of poly(trimethylene terephthalate)-block-poly(tetramethylene oxide) segmented random copolymers. Polym. Adv. Technol. 2011, 22, 72-83. [CrossRef]

12. Liu, F.; Zhang, J.; Wang, J.; Na, H.; Zhu, J. Incorporation of 1,4-cyclohexanedicarboxylic acid into poly(butylene terephthalate)-b-poly(tetramethylene glycol) to alter thermal properties without compromising tensile and elastic properties. RSC Adv. 2015, 5, 94091-94098. [CrossRef]

13. Kint, D.P.R.; Muñoz-Guerra, S. Modification of the thermal properties and crystallization behaviour of poly(ethylene terephthalate) by copolymerization. Polym. Int. 2003, 52, 321-336. [CrossRef]

14. Rosales, A.M.; Murnen, H.K.; Zuckermann, R.N.; Segalman, R.A. Control of crystallization and melting behavior in sequence specific polypeptoids. Macromolecules 2010, 43, 5627-5636. [CrossRef]

15. Zhang, Y.; Feng, Z.; Feng, Q.; Cui, F. Preparation and properties of poly(butylene terephthalate-cocyclohexanedimethylene terephthalate)-b-poly(ethylene glycol) segmented random copolymers. Polym. Degrad. Stab. 2004, 85, 559-570. [CrossRef]

16. Chen, T.; Zhang, J.; You, H. Photodegradation behavior and mechanism of poly(ethylene glycol-co-1,4cyclohexanedimethanol terephthalate) (PETG) random copolymers: Correlation with copolymer composition. RSC Adv. 2016, 6, 102778-102790. [CrossRef]

17. Chen, T.; Jiang, G.; Li, G.; Wu, Z.; Zhang, J. Poly(ethylene glycol-co-1,4-cyclohexanedimethanol terephthalate) random copolymers: Effect of copolymer composition and microstructure on the thermal properties and crystallization behavior. RSC Adv. 2015, 5, 60570-60580. [CrossRef] 
18. Chen, T.; Zhang, J. Surface hydrophilic modification of acrylonitrile-butadiene-styrene terpolymer by poly(ethylene glycol-co-1,4-cyclohexanedimethanol terephthalate): Preparation, characterization, and properties studies. Appl. Surf. Sci. 2016, 388, 133-140. [CrossRef]

19. Rao, Y.Q.; Greener, J.; Avila-Orta, C.A.; Hsiao, B.S.; Blanton, T.N. The relationship between microstructure and toughness of biaxially oriented semicrystalline polyester films. Polymer 2008, 49, 2507-2514. [CrossRef]

20. Jung, I.K.; Lee, K.H.; Chin, I.J.; Yoon, J.S.; Kim, M.N. Properties of biodegradable copolyesters of succinic acid-1,4-butanediol/succinic acid-1,4-cyclohexanedimethanol. J. Appl. Polym. Sci. 2015, 72, 553-561. [CrossRef]

21. Sun, Y.M.; Wang, C.S. Preparation and characterization of poly(ethylene-1,4-cyclohexanedimethylene arylate). Eur. Polym. J. 1999, 35, 1087-1096. [CrossRef]

22. Liu, Y.; Ranucci, E.; Lindblad, M.S.; Albertsson, A.C. New biodegradable polymers from renewable sources: Polyester-carbonates based on 1,3-propylene-co-1,4-cyclohexanedimethylene succinate. J. Polym. Sci. Part A Polym. Chem. 2001, 39, 2508-2519. [CrossRef]

23. Ki, H.C.; Park, O.O. Synthesis, characterization and biodegradability of the biodegradable aliphatic-aromatic random copolyesters. Polymer 2001, 42, 1849-1861. [CrossRef]

24. Celli, A.; Marchese, P.; Sisti, L.; Dumand, D.; Sullalti, S.; Totaro, G. Effect of 1,4-cyclohexylene units on thermal properties of poly(1,4-cyclohexylenedimethylene adipate) and similar aliphatic polyesters. Polym. Int. 2013, 62, 1210-1217. [CrossRef]

25. Bigi, A.; Boanini, E.; Capuccini, C.; Fini, M.; Mihailescu, I.N.; Ristoscu, C.; Sima, F.; Torricelli, P. Biofunctional alendronate-hydroxyapatite thin films deposited by matrix assisted pulsed laser evaporation. Biomaterials 2009, 30, 6168-6177. [CrossRef] [PubMed]

26. Ernault, E.; Richaud, E.; Fayolle, B. Thermal-oxidation of epoxy/amine followed by glass transition temperature changes. Polym. Degrad. Stab. 2017, 138, 82-90. [CrossRef]

27. Vasanthan, N.; Manne, N.J.; Krishnama, A. Effect of molecular orientation on the cold crystallization of amorphous-crystallizable polymers: The case of poly(trimethylene terephthalate). Ind. Eng. Chem. Res. 2013, 52, 17920-17926. [CrossRef]

28. Shi, X.Q.; Aimi, K.; Ito, H.; Ando, S.; Kikutani, T. Characterization on mixed-crystal structure of poly(butylene terephthalate/succinate/adipate) biodegradable copolymer fibers. Polymer 2005, 46, 751-760. [CrossRef]

29. Yoo, H.Y.; Umemoto, S.; Kikutani, T.; Okui, N. Co-crystallization behaviour and melting-point depression in poly(ethylene terephthalate-co-1,4-cyclohexylene dimethylene terephthalate) random copolyesters. Polymer 1994, 35, 117-122. [CrossRef]

30. Bessadok, A.; Roudesli, S.; Marais, S.; Follain, N.; Lebrun, L. Alfa fibres for unsaturated polyester composites reinforcement: Effects of chemical treatments on mechanical and permeation properties. Compos. Part $A$ Appl. Sci. Manuf. 2009, 40, 184-195. [CrossRef]

31. Saint-Loup, R.; Robin, J.J.; Boutevin, B. Synthesis of poly(ethylene terephthalate)- block -poly(tetramethylene oxide) copolymer by direct polyesterification of reactive oligomers. Macromol. Chem. Phys. 2003, 204, 970-982. [CrossRef]

32. Sandhya, T.E.; Ramesh, C.; Sivaram, S. Copolyesters based on poly(butylene terephthalate)s containing cyclohexyl and cyclopentyl ring: Effect of molecular structure on thermal and crystallization behavior. Macromolecules 2007, 40, 6906-6915. [CrossRef]

33. Yamadera, R.; Murano, M. The determination of randomness in copolyesters by high resolution nuclear magnetic resonance. J. Polym. Sci. Part A Polym. Chem. 1967, 5, 2259-2268. [CrossRef]

34. Li, W.D.; Zeng, J.B.; Lou, X.J.; Zhang, J.J.; Wang, Y.Z. Aromatic-aliphatic random and block copolyesters: Synthesis, sequence distribution and thermal properties. Polym. Chem. 2012, 3, 1344-1353. [CrossRef]

35. Matsuda, H.; Nagasaka, B.; Asakura, T. Sequence analysis of poly(ethylene/1,4-cyclohexanedimethylene terephthalate) copolymer using ${ }^{1} \mathrm{H}$ and ${ }^{13} \mathrm{C}$ NMR. Polymer 2003, 44, 4681-4687. [CrossRef]

36. Diao, L.; Su, K.; Li, Z.; Ding, C. Furan-based co-polyesters with enhanced thermal properties: Poly(1,4-butylene-co-1,4-cyclohexanedimethylene-2,5-furandicarboxylic acid). RSC Adv. 2016, 6, 27632-27639. [CrossRef]

37. Zhao, H.B.; Wang, X.L.; Guan, Y.; Wang, X.L.; Chen, L.; Wang, Y.Z. Block self-cross-linkable poly(ethylene terephthalate) copolyester via solid-state polymerization: Crystallization, cross-linking, and flame retardance. Polymer 2015, 70, 68-76. [CrossRef] 
38. Gigli, M.; Lotti, N.; Gazzano, M.; Siracusa, V.; Finelli, L.; Munari, A.; Rosa, M.D. Biodegradable aliphatic copolyesters containing peg-like sequences for sustainable food packaging applications. Polym. Degrad. Stab. 2014, 105, 96-106. [CrossRef]

39. Briber, R.M.; Thomas, E.L. Crystallization behaviour of random block copolymers of poly(butylene terephthalate) and poly(tetramethylene ether glycol). Polymer 1985, 26, 8-16. [CrossRef]

40. Li, R.K.Y.; Tjong, S.C.; Xie, X.L. The structure and physical properties of in situ composites based on semiflexible thermotropic liquid crystalline copolyesteramide and poly(butylene terephthalate). J. Polym. Sci. Part B Polym. Phys. 2000, 38, 403-414. [CrossRef]

41. Boye, C.A. X-ray diffraction studies of poly(1,4-cyclohexylenedimethylene terephthalate). J. Polym. Sci. 1961, 55, 275-284. [CrossRef]

42. Han, S.; Kim, C.; Kwon, D. Thermal degradation of poly(ethyleneglycol). Polym. Degrad. Stab. 1995, 47, 203-208. [CrossRef]

43. Lotti, N.; Colonna, M.; Fiorini, M.; Finelli, L.; Berti, C. Poly(butylene terephthalate) modified with ethoxylated bisphenol s with increased glass transition temperature and improved thermal stability. Polymer 2011, 52, 904-911. [CrossRef]

(C) 2017 by the authors. Licensee MDPI, Basel, Switzerland. This article is an open access article distributed under the terms and conditions of the Creative Commons Attribution (CC BY) license (http:/ / creativecommons.org/licenses/by/4.0/). 